\title{
Long-term functional recovery after orthotopic spiral ileal bladder substitution
}

\author{
Xiaonong Chen, Weigao Wang, Anping Xiang, Yuehong Li \\ Department of Urology, The First People's Hospital of Huzhou, The First Affiliated Hospital of Huzhou Teachers College, Huzhou 313000 , China \\ Contributions: (I) Conception and design: X Chen; (II) Administrative support: W Wang; (III) Provision of study materials: A Xiang; (IV) Collection \\ and assembly of data: X Chen, A Xiang; (V) Data analysis and interpretation: X Chen, W Wang; (VI) Manuscript writing: All authors; (VII) Final \\ approval of manuscript: All authors. \\ Correspondence to: Yuehong Li. Department of Urology, The First People's Hospital of Huzhou, The First Affiliated Hospital of Huzhou Teachers \\ College, Huzhou 313000, China. Email: lyh19821123@163.com.
}

Background: To explore the value of orthotopic spiral ileal bladder substitution (OSIBS) following radical cystectomy in treating bladder cancer patients by investigating the short- and long-term postoperative complications and assessing the quality of life in patients who had undergone OSIBS.

Methods: The post-operative complications were retrospectively analyzed among bladder cancer patients who had undergone radical cystectomy + OSIBS in our center from January 2001 to January 2017. The quality of life was assessed by using the Function Assessment of Cancer Therapy-Bladder Cancer Form (FACT-BL). Patients were followed up by mails, telephone, and outpatient visits.

Results: A total of 68 subjects were included in this study. All patients undergoing radical cystectomy + OSIBS were followed up for an extended period. The patients aged 39-68 years (mean: 60.34 9.43 years). The surgeries were completed, and all the patients were smoothly discharged after good postoperative recovery. Of the 68 patients who had completed the follow-up visits, 10 had early complications (14.71\%), and $11(17.64 \%)$ developed late complications. The blood urea nitrogen (BUN) $(t=0.358, P=0.764)$ and serum creatinine $(\mathrm{Cr})(\mathrm{t}=1.305, \mathrm{P}=0.196)$ levels showed no significant difference before and after surgery. The serum potassium ( $\mathrm{t}=1.347, \mathrm{P}=0.169)$, sodium ( $\mathrm{t}=-1.748, \mathrm{P}=0.144)$, and calcium ( $\mathrm{t}=1.097, \mathrm{P}=0.319)$ levels also showed no significant changes before and after surgery. However, the change in serum chlorine level was statistically significant $(\mathrm{t}=-4.701, \mathrm{P}=0.000)$. To support urinary function, the patients were encouraged to take exercises During the 10-year follow-up period, the daytime urinary continence rate reached $94.1 \%$ ( $n=64)$ in the daytime, and the nighttime incontinence rate was $13.2 \%(\mathrm{n}=9)$. Six months after the surgery, the neobladder capacity was $(365.02 \pm 45.11) \mathrm{mL}$, the maximum flow rate was $(14.36 \pm 1.41) \mathrm{mL} / \mathrm{s}$, and the postvoiding residual $(\mathrm{PVR})$ was $(26.01 \pm 8.10) \mathrm{mL}$. The total FACT-BL score was $(124.8 \pm 13.4)$ during the 10 -year follow-up.

Conclusions: After 10 years of follow-up, the early and late complications in patients who had undergone radical cystectomy + OSIBS were within acceptable range and the daily and nightly continence levels allowed normal daily life. OSIBS not only preserves the physical integrity of the body but also has normal physiological characteristics of the bladder. It remarkably improves the postoperative quality of life and can be easily accepted by patients. Therefore, it is an ideal surgical procedure.

Keywords: Bladder cancer; radical cystectomy; Orthotopic spiral ileal bladder substitution (OSIBS); Function Assessment of Cancer Therapy-Bladder Cancer Form (FACT-BL); functional evaluation

Submitted Dec 26, 2019. Accepted for publication Feb 25, 2020.

doi: $10.21037 /$ tau.2020.03.16

View this article at: http://dx.doi.org/10.21037/tau.2020.03.16 


\section{Introduction}

Orthotopic urinary diversion after total bladder resection has become the mainstream bladder substitution technique due to its satisfactory clinical effectiveness, high postoperative quality of life, and low incidences of complications. In our current study, we retrospectively analyzed the clinical data of 68 patients who had undergone radical cystectomy + orthotopic spiral ileal bladder subs titution (OSIBS) for bladder cancer in our center between January 2001 and January 2017. Both the early and late complications were within the acceptable ranges. The functions of the neobladder in urine storage and urine expulsion were close to the physiological states, and thus OSIBS is an ideal surgical procedure.

\section{Methods}

\section{General data}

\section{Subjects}

Totally 68 patients diagnosed with bladder cancer who underwent total bladder resection + OSIBS in our hospital from January 2001 to January 2017 were selected as the research subjects. The Ethics committee approved this study of our center. The surgical results were satisfactory, and all the patients were followed up for an extended period. The patient's name, medical record number, gender, age, contact address and phone number, clinical manifestations, date of surgery, complications, postoperative biochemistry, urinary tract imaging findings, and urodynamic examination results were recorded. These patients included 30 men and two women aged $39-79$ years $[(60.34 \pm 9.43)$ years]. The hospital stay was $(38.45 \pm 13.33)$ days. Among these patients, 34 had gross hematuria, and 10 experienced frequent and painful urination. The disease recurred after transurethral resection of the bladder tumor in one case. All patients received longterm follow-up after operation. The follow-up ranged from 3 to 120 months, with an average of 12.9 months. Before the surgery, the diagnoses were confirmed after ultrasound and CT of the urinary system and cystoscopy. Postoperative pathology revealed 66 cases of urothelial carcinoma ( 2 cases in $T_{1}$ stage, 44 cases in $T_{2}$ stage, and 20 cases in $T_{3}$ stage) and 1 case of adenocarcinoma. The diagnoses were confirmed by postoperative pathological examination in all the 68 cases.

\section{Inclusion criteria}

(I) With pathologically confirmed bladder cancer; (II) with primary bladder cancer; (III) without procedure-related major complication; (IV) in good mental state and with good communication skills; and (V) can be interviewed via telephone.

\section{Exclusion criteria}

(I) Accompanied by damage to vital organs or other serious chronic diseases; (II) with a prior history of mental illness or with severe cognitive impairment that makes the patient unable to cooperate during the communication; and (III) patients refused to join the study after the purpose, method, and significance of the study were explained to the patients and their families.

\section{Surgical methods}

\section{Pre-operative preparation}

Patients were carefully evaluated before surgery. Blood pressure and blood glucose were actively adjusted. Electrolyte disorders, anemia, hypoproteinemia, and other conditions were corrected. Intestinal preparation was started three days before the operation, and metronidazole + gentamicin was administered orally to inhibit the growth of gut flora. During the 3-day preparation, semi-liquid diets were given on the first day, liquid diets on the second day, and fasting and total parenteral nutrition on the third day. Cleaning enemas were administered the night before surgery and on the operation day, and metronidazole retention enema was given before the operation.

\section{Anesthesia and body position}

The combination of intravenous and inhaled anesthesia was routinely used. The patient was asked to take a supine position, with a raised hip by a cushion and a head-feet angle of about $10^{\circ}-15^{\circ}$. A urinary catheter (F16-F18) was placed. A midline incision was created in the lower abdomen, via which the abdominal wall was cut open layer by layer. The abdominal organs were systematically explored for any tumor metastasis. A blunt separation of the retropubic space was performed along the pubic bone downwards the pelvic floor for standard radical cystectomy (along with removal of prostate and seminal vesicles in males and resection of uterus, appendages, and part of the anterior vaginal wall in females); meanwhile, lymph nodes around the obturator nerves and the vessels in iliac crest were routinely dissected. After the abdominal cavity was opened, about $40-45 \mathrm{~cm}$ of ileum about $30 \mathrm{~cm}$ away from the ileocecal area was transected to restore intestinal continuity. The extracted 


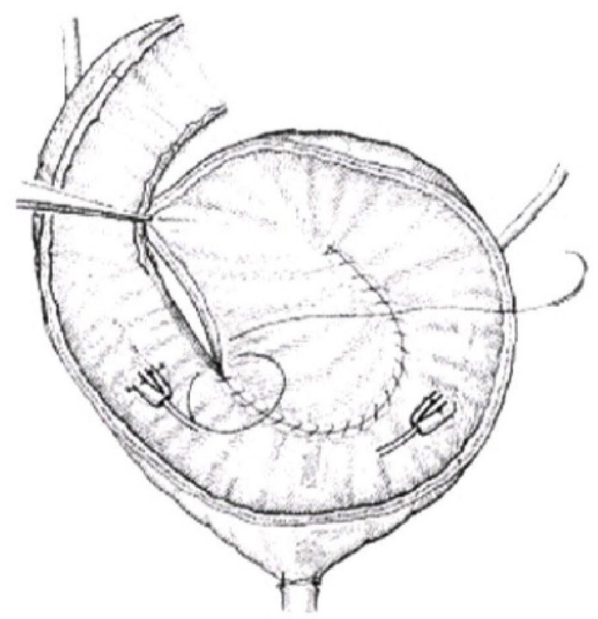

Figure 1 The isolated ileal loop forms spiral urinary reservoir.

intestinal tube was repeatedly irrigated and disinfected with dilute solutions of povidone-iodine and then cut open along the mesentery's opposite side. The intestinal segment was scraped with a suction device to remove mucus on the surface of the intestinal mucosa and repeatedly wiped with absolute ethanol to destroy the mucous cells on the intestinal surface. The treated ileal segment was spirally folded and then closed with a continuous full-layer blanket using 2-0 absorbable sutures to form a sphere-shaped lowpressure urinary reservoir (Figure 1). The ends of bilateral ureters were divided by $1.0 \mathrm{~cm}$ and pulled outwards for trimming, showing a cuff-shaped nipple. The intestinal wall was divided in situ to create a 5 -cm hole, and the newlycreated urinary reservoir was inserted by $1.0 \mathrm{~cm}$ to form a small nipple (the split-cuff ureteral nipple), which played an anti-reflux role. The lowest end of the neobladder was divided by $1.0 \mathrm{~cm}$, and 4 stitches were intermittently made with full-thickness 2-0 absorbable sutures at the corresponding sites $(2,5,7$, and 10$)$. A three-way balloon urethral catheter was placed into the new urinary reservoir and gently pulled for $24 \mathrm{~h}$ to reduce the anastomotic tension. A single jejunostomy tube (J-tube), which was placed inside the ureter, and a neobladder ostomy tube were introduced through the abdominal wall, along with a low drainage tube placed at the pelvic wound. The drainage tube was removed 3 to 5 days after the operation. One week later, $100 \mathrm{~mL}$ of metronidazole solution and $100 \mathrm{~mL}$ of $5 \%$ sodium bicarbonate solution was dripped into the neobladder daily. The J-tube in the ureter was removed 7 to 10 days later, and the neobladder ostomy tube was withdrawn two weeks later. The urinary catheter was removed 16 to 21 days after surgery to restore urethral urination.

\section{Research methods}

The clinical, biochemical, imaging and urodynamic data of patients undergoing radical cystectomy + OSIBS in our center from January 2001 to January 2017 were retrospectively analyzed. The patients were followed up for 3 to 120 months, and the quality of life of these patients was assessed by using the Function Assessment of Cancer Therapy-Bladder Cancer Form (FACT-BL).

FACT was developed by Celia et al. of Luke Medical Center. This system consists of a general scale (FACT-G) and several cancer-specific subscales. The third edition of FACT-G includes 34 items covering five domains: physical well-being (8 items), social/family well-being (8 items), relationship with doctors (3 items), emotional well-being (7 items), and functional well-being (7 items). The last item in each domain is an overall evaluation of this domain (for total assessment and weighted scoring), which is not included in the calculation of the score for each domain. The fourth edition of the FACT-G used in our current study deleted the last item of each domain, as seen in the original scale, and the entire domain of "relationship with doctors", yielding 27 items; after 12 items in the bladder cancer-specific subscale were added, a total of 39 items were used in our current study.

\section{Statistical analysis}

Statistical analyses were performed using the SPSS 19.0 software package. Measurement data are expressed as $\bar{x} \pm$ SD and compared with a $t$-test. A comparison of count data was performed using the chi-square test. A $\mathrm{P}$ value of less than 0 was considered statistically significant.

\section{Results}

A total of 68 subjects were included in this study. All patients underwent radical cystectomy + OSIBS and were followed up for an extended period. These patients included 64 men and 4 women aged $39-79$ years [(60.34 \pm 9.43$)$ years]. The hospital stay was $(38.45 \pm 13.33)$ days (Table 1$)$.

\section{Postoperative complications}

The short-term postoperative complications mainly included: (I) incomplete intestinal obstruction $(\mathrm{n}=4)$, which 
Table 1 General data of participants and non-participants

\begin{tabular}{lccc}
\hline General data & Participants & Non-participants & P value \\
\hline $\mathrm{n}$ & 68 & 25 & - \\
Males (\%) & $64(94.14)$ & $23(92.00)$ & $>0.05$ \\
Age (years) & $57.4 \pm 9.3$ & $58.2 \pm 10.3$ & $>0.05$ \\
Education (years) & $12.1 \pm 4.2$ & $11.5 \pm 4.1$ & $>0.05$ \\
Surgical time (hours) & $3.4 \pm 0.5$ & $3.8 \pm 0.4$ & $>0.05$ \\
Hospital stay (days) & $22.3 \pm 2.9$ & $21.8 \pm 3.1$ & $>0.05$ \\
\hline
\end{tabular}

Table 2 Post-operative complications

\begin{tabular}{lll}
\hline Period of complications & Post-operative complications & $\mathrm{n}$ \\
\hline Early complications & Urinary leakage & 4 \\
& Intestinal obstruction & 4 \\
& Poor incision healing & 2 \\
Late complications & Urinary incontinence & 8 \\
& Metabolic disorder & 0 \\
& Renal function impairment & 0 \\
& Urethral tumor recurrence & 1 \\
& Neobladder stones & 2 \\
Total & & 21 \\
\hline
\end{tabular}

was improved after conservative treatments including gastrointestinal decompression and acid suppression; (II) urinary leakage $(\mathrm{n}=4)$, which included 2 cases of neobladder and urethral anastomotic leakage, which were cured by conservative treatments such as proper traction of urinary catheter and prolonged indwelling time, and 2 cases of urethral-neobladder anastomotic leakage, which was cured after surgical intervention; and (III) fat liquefaction and poor incision healing $(n=2)$, which were cured after conservative treatment. The long-term postoperative complications mainly included: urinary incontinence ( $\mathrm{n}=8$, including 4 females and 4 males); neobladder stones $(\mathrm{n}=2)$; and urethral tumor recurrence $(\mathrm{n}=1)$ (Table 2).

Of the 68 patients who had completed the follow-up visits, 46 had no complications $(67.65 \%)$, and 21 suffered from complications (32.35\%). Among them, ten experienced early complications, and 11 had late complications. Three patients required surgical intervention.

\section{Urinary continence}

All patients stood on their own to void without postoperative catheterization. After urinary extubation, most patients had transient mild urinary incontinence. Eight patients, including four males and four females, suffered from urinary incontinence. All four female patients had genuine incontinence. The four male patients adopted the anuslifting exercise and changed their urination habits; their urinary incontinence was slightly improved after three months and remarkably improved after six months. The urination and urinary continence were good in most of these 64 patients: 64 patients $(94.1 \%)$ achieved urinary continence in the daytime, and nine patients (13.2\%) had nocturnal urinary incontinence. The patients voided 4-8 times in daytime and 2-5 times at night, with the void volume being $150-300 \mathrm{~mL}$ and the voiding interval being $2-5$ hours. The volume of 24 -hour water intake was $600-2,400 \mathrm{~mL}$. The urination frequency gradually decreased, and the volume of each urination gradually increased after surgery. The urinary continence was regained after anus-lifting exercise and pelvic floor muscle training in 60 patients.

\section{Urodynamics}

Urodynamic tests performed six months after the surgery. After bladder function training after the surgery, the neobladder capacity was significantly higher in the six post-operative months than in the $3 \mathrm{rd}$ postoperative month $(\mathrm{P}<0.05)$ and the PVR was significantly smaller $(\mathrm{P}<0.05)$; although the maximum flow rate was higher in the six post-operative months than in the $3 \mathrm{rd}$ postoperative month, the difference was not statistically significant $(\mathrm{P}>0.05)$ (Table 3). 
Table 3 Urodynamics 3 and 6 months after surgery

\begin{tabular}{lcccc}
\hline Item & 3 months after surgery & 6 months after surgery & t value & P value \\
\hline Neobladder capacity $(\mathrm{mL})$ & $285.03 \pm 35.01$ & $365.02 \pm 45.11$ & -3.050 & 0.002 \\
Post-voiding residual $(\mathrm{mL})$ & $45.11 \pm 9.02$ & $26.01 \pm 8.10$ & -5.568 & 0.001 \\
Maximum flow rate $(\mathrm{mL} / \mathrm{s})$ & $10.71 \pm 1.32$ & $11.36 \pm 1.41$ & -0.850 & 0.050 \\
\hline
\end{tabular}

Table 4 Comparison of renal function before and after surgery

\begin{tabular}{lccc}
\hline Comparison of renal function & $\mathrm{n}$ & Serum Cr $(\mu \mathrm{mol} / \mathrm{L})$ & $\mathrm{BUN}(\mu \mathrm{mol} / \mathrm{L})$ \\
\hline Before surgery & 68 & $102.71 \pm 18.61$ & $5.79 \pm 1.57$ \\
After surgery & 68 & $106.24 \pm 21.33$ & $6.01 \pm 1.87$ \\
Difference & & $3.53 \pm 20.18$ & $6.01 \pm 1.87$ \\
$\mathrm{t}$ value & & 1.305 & 0.358 \\
$\mathrm{P}$ value & & 0.196 & 0.764 \\
\hline
\end{tabular}

BUN, blood urea nitrogen.

Table 5 Comparison of electrolytes before and after surgery

\begin{tabular}{lccccc}
\hline Comparison of electrolytes & $\mathrm{N}$ & $\mathrm{K}^{+}(\mathrm{mmol} / \mathrm{L})$ & $\mathrm{Na}^{+}(\mathrm{mmol} / \mathrm{L})$ & $\mathrm{Ca}^{+}(\mathrm{mmol} / \mathrm{L})$ & $\mathrm{Cl}^{-}(\mathrm{mmol} / \mathrm{L})$ \\
\hline Before surgery & 68 & $4.33 \pm 0.27$ & $139.57 \pm 2.65$ & $2.40 \pm 0.04$ & $105.28 \pm 0.26$ \\
After surgery & 68 & $4.28 \pm 0.17$ & $140.47 \pm 2.94$ & $2.37 \pm 0.07$ & $0.03 \pm 0.12$ \\
Difference & & $0.05 \pm 1.13$ & $-0.90 \pm 3.01$ & 1.097 & $-0.52 \pm 0.47$ \\
$t$ value & 1.347 & 0.169 & 0.144 & 0.319 & -1.701 \\
P value & & & 0.154 & 0.48 \\
\hline
\end{tabular}

Table 6 Scores of different domains based on FACT-BL

\begin{tabular}{ll}
\hline Domain (total score) & Scores \\
\hline Physical well-being & $24.2 \pm 3.2$ \\
Social/family well-being & $23.5 \pm 2.5$ \\
Emotional well-being & $20.2 \pm 1.5$ \\
Functional well-being & $23.9 \pm 2.1$ \\
FACT-G (108) & $92 \pm 9.4$ \\
Bladder cancer-specific template (48) & $32.8 \pm 4.1$ \\
FACT-BL (156) & $124.8 \pm 13.4$ \\
\hline
\end{tabular}

FACT-BL, Function Assessment of Cancer Therapy-Bladder Cancer Form; FACT-G, Function Assessment of Cancer TherapyGeneric Scale.

\section{Renal function}

Twelve months after the operation, the serum Cr level and the serum BUN (Table 4).

\section{Electrolytes}

Twelve months after the operation, the serum electrolyte levels in these 68 patients (Table 5).

\section{Scores of quality of life}

Table 6 summarizes the scores of quality of life 24 months after surgery (based on the FACT-BL scale).

As shown in the above table, the satisfaction rate of urinary continence, the appearance of my body, and sexual life was $80.88 \%(55 / 68), 88.24 \%(60 / 68)$, and $10.29 \%$ (7/68), respectively. Eight patients (12.50\%) experienced erection problems. Besides, when asked if they have the opportunity to choose the surgical procedure again, $88.24 \%(60 / 68)$ of the respondents still chose the current surgical method, indicating that these patients, as a whole, were then satisfied with the current surgical treatment (Table 7). 
Table 7 Distribution of bladder cancer-specific template

\begin{tabular}{lcrrr}
\hline Satisfaction and urinary control & Never & Occasionally & Sometimes & Often \\
\hline I have trouble controlling my urine & 55 & 8 & 4 & 1 \\
I urinate more frequently than usual & 14 & 22 & 22 & 6 \\
I like the appearance of my body & 0 & 0 & 4 & 12 \\
I am interested in sex & 7 & 10 & 43 & 0 \\
(For men only) I can have and maintain an erection & 8 & 10 & 40 & 16 \\
\hline
\end{tabular}

\section{Urinary continence}

Urinary continence following OSIBS directly affects the quality of life. A significant advantage of OSIBS is that the function of the neobladder in urine expulsion is close to the physiological state. Therefore, the urinary continence rate has become the primary criterion for evaluating the value of OSIBS. A recent consensus on the effectiveness of OSIBS in urinary continence is that OSIBS can achieve good urinary continence in the daytime, but the efficiency is relatively poor at night. In our current study, the urinary continence rate was $94.1 \%$ and $86.8 \%$, respectively, in the daytime and at night in 68 patients who had undergone OSIBS.

\section{Discussion}

Orthotopic bladder substitution has developed rapidly due to its advantages, including autonomous urination, good urinary continence, no need for the abdominal stoma, and improved quality of life $(1,2)$. It has become the mainstream urinary diversion technique in some critical medical centers abroad (3), and a study with a large sample size has also been available in China (4). Urinary diversion is advancing from mere diversion of urine to the outside of the body via the intestinal canal to the diversion that mimics the physiological urination. An ideal neobladder should meet the following requirements: (I) basically in line with the physiological excretion function of the human body; (II) able to store urine under low pressure $\left(\leq 40 \mathrm{cmH}_{2} \mathrm{O}\right.$ when filled); (III) with adequate capacity (up to $300-500 \mathrm{~mL}$ ) and proper compliance; (IV) without or with only mild water-electrolyte imbalance and relatively stable internal environment; (V) without ureteral reflux and thus without causing damage to the renal function; and (VI) without or with only mild ascending infections in urinary tract. In 2001, Beck and Koch (5) described the spiral ileal neobladder substitution, which was considered to have the advantages of high intestinal utilization and satisfactory postoperative urine continence. Compared with Studer's U-shaped surgical procedure (6), the spiral ileal neobladder substitution was theoretically more spherical and required less intestinal canal at the same volume, thus had less interference on the digestive tract; since the intestinal longitudinal muscles were preserved, the neobladder could function under relatively low pressure, which increased the compliance of the neobladder. These advantages were also validated in our current study.

Technical improvements in our center include: (I) for the intestinal wall of the neobladder, scraping and suction method was used to remove mucus, and absolute ethanol was used to treat mucous membranes. It remarkably reduces the postoperative secretion of intestinal mucus and lowers the postoperative incidences of mucus obstruction in the urinary tract and absorption-related metabolic disorders; (II) the ureter is inserted orthotopically, which avoids ureteral stricture and renal reflux dysfunction caused by ischemic necrosis and twisting of the distal ureter following excessive mobilization; (III) the four-stitch technique for suturing the neobladder and urethra makes the operation time-saving and straightforward and reduces the occurrence of anastomotic stenosis. Intraoperative handling of the neobladder is particularly crucial for lowering the mucous secretion of the neobladder and preventing the subsequent absorption-related metabolic disorders. Studies have shown that there is more mucus secreted by the intestinal mucosa in the early postoperative period. Due to the influence of urine, the intestinal mucosa will gradually experience some changes, which is featured by inflammation in the first stage, followed by the degenerative phase, during which tissues such as the brush border and microvilli will be destroyed and disappear, and their absorption capacity will eventually decrease by eight times. The mucosal epithelium will finally have functions, including coverage and protection, just as urinary epithelium does. 
Wang et al. (7) treated the intestinal mucosa with $95 \%$ ethanol to inactivate its functional cells. The longterm storage of urine caused changes in the structure and function of the intestinal mucosa, lowering their absorption and secretion functions. After the disinfected intestinal canal was cut open, the mucus on the surface of the intestinal mucosa was removed with a suction device to make the scraping with absolute ethanol more effective. After the above treatments, it was found that the mucus secretion in the neobladder was significantly reduced in the early stage, and no complications such as hypernatremia, hyperchloremia, and atrophy or fibrosis of the neobladder were observed during postoperative follow-up.

According to our experience, an excessively wide pedicled mesentery will reduce the mobilizability of the intestinal canal and increase the difficulty in spiralforming. The traction and compression of the neobladder by mesentery will restrict the filling of the neobladder and reduce its capacity. Therefore, the width of the mesentery should be minimized while ensuring good blood supply. A 1-cm "plug-in" nipple of the ureter may be feasible to ensure the blood supply of the nipple, mucosal coverage, and prevention of nipple retraction. The neobladderurethral anastomosis is another critical step in the surgery. We used the posterior urethral water-bag to fix the urethral catheter, which was pulled towards the pelvic cavity to completely expose the urethra and ease the eversion of the mucosa. Thus, the anastomosis between urethra and neobladder became simple, reliable, and time-saving. In our current study, the urine volume after extubation reached about $200 \mathrm{~mL}$, with a void interval of 1-2 hours. Urodynamic examinations showed that the pressure of the "detrusor muscle" of the neobladder was relatively low. The bladder capacity for the first voiding was $315 \mathrm{~mL}$, and the maximum bladder capacity was $600 \mathrm{~mL}$. Thus, treatment effectiveness was satisfactory. Also, urinary continence has become a key indicator for evaluating surgical effectiveness. Many international studies have reported that a variety of neobladder procedures could achieve good urinary continence both in the daytime and at night (8-11). Steers et al. (12) analyzed the data of 2,238 patients undergoing neobladder operations and found the incidence of urinary incontinence was only $13.3 \%$ to $13.6 \%$. In our current study, the rate of urine continence was $94.1 \%(n=64)$ in the daytime and $86.8 \%(\mathrm{n}=59)$ at night, which was comparable to the results of other large-scale studies.
In summary, OSIBS following the radical cystectomy has the following advantages: (I) under the contrast-enhanced $\mathrm{X}$-ray, the shape of the neobladder is more spherical when filled and has larger capacity; (II) it has relatively low internal pressure and thus the compliance is proper; (III) the harvested intestinal canal is short and therefore poses smaller interference to the GI tract; (IV) the function of urination is regained early after surgery, and the long-term urination continence in daytime and at night is satisfactory; (V) the treated new bladder has significantly fewer postoperative mucus secretions and less urine absorption during urinary storage, which prevent postoperative urinary tract obstruction and metabolic disorders; (VI) the "plugin" nipple method for neobladder is simple and has low tension, which effectively prevents urine reflux and ureteral stricture, thereby protecting renal function; and (VII) the "four-stitch method" used in anastomosis is practical and straightforward, lowering the incidence of neobladderurethral anastomotic stricture.

Therefore, OSIBS is a neobladder reconstruction technique that is more in line with physiological states. Few patients developed water-electrolyte balance disturbance, and the upper urinary tract function was restored well. The features of the neobladder in urine storage and urine expulsion were close to the physiological states. OSIBS not only preserves the physical integrity of the body but also has typical physiological characteristics of the bladder. It remarkably improves the postoperative quality of life and can be readily accepted by patients. Therefore, it is an ideal surgical procedure.

\section{Acknowledgments}

Funding: None.

\section{Footnote}

Conflicts of Interest: The authors have no conflicts of interest to declare.

Ethical Statement: The authors are accountable for all aspects of the work in ensuring that questions related to the accuracy or integrity of any part of the work are appropriately investigated and resolved. The Ethics committee approved this study of our center (No. 2017003). The surgical results were satisfactory, and all the patients were followed up for an extended period. 
Open Access Statement: This is an Open Access article distributed in accordance with the Creative Commons Attribution-NonCommercial-NoDerivs 4.0 International License (CC BY-NC-ND 4.0), which permits the noncommercial replication and distribution of the article with the strict proviso that no changes or edits are made and the original work is properly cited (including links to both the formal publication through the relevant DOI and the license). See: https://creativecommons.org/licenses/by-nc$\mathrm{nd} / 4.0 /$.

\section{References}

1. Weijerman PC, Schurmans JR, Hop WC, et al. Morbidity and quality of life in patients with orthotopic and heterotopic continent urinary diversion. Urology 1998;51:51-6.

2. Sullivan LD, Chow VD, Ko DS, et al. An evaluation of quality of life in patients with continent urinary diversions after cystectomy. Br J Urol 1998;81:699-704.

3. Hautmann RE. Urinary diversion: ileal conduit to neobladder. J Urol 2003;169:834-42.

4. Ye M. Technical refinements and clinical application of Studer neobladder. Chinese Journal of Urology 2003;24:686-90.

Cite this article as: Chen X, Wang W, Xiang A, Li Y. Longterm functional recovery after orthotopic spiral ileal bladder substitution. Transl Androl Urol 2020;9(2):665-672. doi: $10.21037 /$ tau.2020.03.16
5. Beck SD, Koch MO. Spiral ileal neobladder substitution with orthotopic ureteral reimplantation: $\infty$ rly results. Tech Urol 2001;7:223-8.

6. Studer UE, Varol C, Danu8er H.Orthotopderi ileal neobladder. BJU Int 2004;93:183-93.

7. Wang LL, Wang TC, Gao X. Experimental study on the inactivation of intestinal functional cells by absolute ethanol. Chinese Journal of Urology 1992;13:91-4.

8. Abol-Enein H, Ghoneim MA. Functional results of orthotopic ileal neobladder with serous-lined extramural ureteral reimplantation: experience with 450 patients. J Urol 2001;165:1427-32.

9. Hautmann RE. De Petricioni R, Gottfried HW, et al. The ileal neobladder: complications and functional results in 363 patients after 11 years of follow up. J Urol 1999;161:422-7; discussion 427-8.

10. Hollowell CM, Christiano AP, Steinberg GD. Technique of Hautmann ileal neobladder with chimney modification: interim results in 50 patients. J Urol 2000;163:47-50; discussion 50-1.

11. Studer UE, Zingg EJ. Ileal orthotopic bladder substitutes. What we have learned from 12 years' experience with 200 patients. Urol Clin North Am 1997;24:781-93.

12. Steers WD. Voiding dysfunction in the orthotopic neobladder. World J Urol 2000;18:330-7. 